\title{
Universal spaces in the theory of transfinite dimension, I
}

\author{
by
}

Wojciech O lszewski (Warszawa)

\begin{abstract}
R. Pol has shown that for every countable ordinal $\alpha$, there exists a universal space for separable metrizable spaces $X$ with ind $X=\alpha$.

We prove that for every countable limit ordinal $\lambda$, there is no universal space for separable metrizable spaces $X$ with Ind $X=\lambda$. This implies that there is no universal space for compact metrizable spaces $X$ with Ind $X=\lambda$. We also prove that there is no universal space for compact metrizable spaces $X$ with ind $X=\lambda$.
\end{abstract}

1. Introduction. Our terminology and notation follow the books [3] and [1], with the exception of the boundary, closure and interior of a subset $A$ of a space $X$, which are denoted by bd $A, \operatorname{cl} A$ and int $A$, respectively, and the diameter of a subset $A$ of a metric space $(X, \varrho)$, which is denoted by $\operatorname{diam} A$ or $\operatorname{diam}_{\varrho} A$. If $Y$ is a subspace of $X$, and $A$ is contained in $Y$, then the boundary, closure and interior of $A$ in $Y$ are denoted by $\operatorname{bd}_{Y} A, \operatorname{cl}_{Y} A$ and $\operatorname{int}_{Y} A$, respectively.

The transfinite dimensions ind and Ind are transfinite extensions of the classical Menger-Urysohn small inductive dimension ind and Brouwer-Čech large inductive dimension Ind, respectively.

1.1. Definition (see [4]). Let $X$ denote a regular space, and let $\alpha$ be the integer -1 , an ordinal, or the symbol $\infty$. The following conditions define the small transfinite dimension of $X$ :

(1.1) ind $X=-1$ if and only if $X=\emptyset$,

(1.2) ind $X \leq \alpha \geq 0$ if for every $x \in X$ and every neighbourhood $V \subseteq X$ of $x$, there exists an open set $U \subseteq X$ such that $x \in U \subseteq V$ and ind $\operatorname{bd} U<\alpha$ (i.e., ind $\mathrm{bd} U \leq \beta$ for a $\beta<\alpha$ ),

(1.3) ind $X=\alpha$ if ind $X \leq \alpha$ and the inequality ind $X<\alpha$ does not hold,

(1.4) ind $X=\infty$ if ind $X>\alpha$ (i.e., the inequality ind $X \leq \alpha$ does not hold) for every ordinal $\alpha$.

1991 Mathematics Subject Classification: Primary 54F45. 
We say that the small transfinite dimension of a regular space $X$ at a point $x$ does not exceed an ordinal $\alpha$, and we write $\operatorname{ind}_{x} X \leq \alpha$, if for every neighbourhood $V \subseteq X$ of $x$, there exists an open set $U \subseteq X$ such that $x \in U \subseteq V$ and ind bd $U<\alpha$. If $\operatorname{ind}_{x} X \leq \alpha$ and $\operatorname{ind}_{x} X>\beta$ for every $\beta<\alpha$, then we say that the small transfinite dimension of $X$ at $x$ is $\alpha$, and we write $\operatorname{ind}_{x} X=\alpha$. We say that the small transfinite dimension of $X$ at $x$ is $\infty$ if $\operatorname{ind}_{x} X>\alpha$ for every ordinal $\alpha$.

It is easy to check that ind $X \leq \alpha$ if and only if $\operatorname{ind}_{x} X \leq \alpha$ for every $x \in X$.

1.2. Definition (see [16]). Let $X$ denote a normal space, and let $\alpha$ be the integer -1 , an ordinal, or the symbol $\infty$. The following conditions define the large transfinite dimension of $X$ :

(1.5) Ind $X=-1$ if and only if $X=\emptyset$,

(1.6) Ind $X \leq \alpha \geq 0$ if for every closed set $A \subseteq X$ and every open set $V \subseteq X$ which contains $A$, there exists an open set $U \subseteq X$ such that $A \subseteq U \subseteq V$ and Ind bd $U<\alpha$,

(1.7) Ind $X=\alpha$ if Ind $X \leq \alpha$ and the inequality Ind $X<\alpha$ does not hold,

(1.8) Ind $X=\infty$ if Ind $X>\alpha$ for every ordinal $\alpha$.

Observe that if we replace the ordinal $\alpha$ by a natural number $n$ in Definitions 1.1 and 1.2, then we obtain the definitions of the small inductive dimension of Menger-Urysohn and of the large inductive dimension of Brouwer-Čech.

Let $X$ be a topological space and $A, B$ a pair of disjoint subsets of $X$; we say that a set $L \subseteq X$ is a partition (in $X$ ) between $A$ and $B$ if there exist open sets $U, V \subseteq X$ satisfying:

$$
A \subseteq U, \quad B \subseteq V, \quad U \cap V=\emptyset \quad \text { and } \quad X-L=U \cup V .
$$

One can check that a regular space $X$ (a normal space $X$ ) satisfies ind $X \leq \alpha$ (Ind $X \leq \alpha)$ if and only if for every $x \in X$ and each closed set $B \subseteq X$ such that $x \notin B$ (resp. for every pair $A, B$ of disjoint closed subsets of $X$ ) there exists a partition $L$ between $x$ and $A$ (a partition $L$ between $A$ and $B$ ) such that ind $L<\alpha$ (Ind $L<\alpha$ ).

For simplicity of notation we assume that $\alpha<\infty, \alpha+\infty=\infty+\alpha=\infty$, and $\alpha \cdot \infty=\infty \cdot \alpha=\infty$ for every ordinal $\alpha$.

If $X$ is a separable metrizable space and $-1<$ ind $X<\infty(-1<$ Ind $X$ $<\infty)$, then ind $X$ (Ind $X)$ is a countable ordinal, i.e., ind $X<\omega_{1}$ (Ind $X<$ $\omega_{1}$ ) (see [4] and [16], or [2], Theorems 3.5 and 3.8). Obviously, ind $X \leq \operatorname{Ind} X$ for every normal space $X$, but the inverse inequality does not hold; there exists a compact metrizable space $X$ such that ind $X<\operatorname{Ind} X$ (see [9]). For every ordinal $\alpha$, there exist compact metrizable spaces $X$ and $Y$ such that 
ind $X=\alpha$ and Ind $Y=\alpha$ (see Section 2). For a deeper discussion we refer the reader to R. Engelking's survey [2].

We shall only be concerned with the question of universal spaces. Let us recall that a space $X$ is universal in a class $\mathcal{C}$ of spaces if $X$ belongs to $\mathcal{C}$ and every space of $\mathcal{C}$ is embeddable in $X$. The following questions are natural in this context ( $\alpha$ is a countable ordinal).

1. Does there exist a universal space for compact metrizable spaces $X$ with ind $X \leq \alpha$ ?

2. Does there exist a universal space for compact metrizable spaces $X$ with Ind $X \leq \alpha$ ?

3. Does there exist a universal space for separable metrizable spaces $X$ with ind $X \leq \alpha$ ?

4. Does there exist a universal space for separable metrizable spaces $X$ with Ind $X \leq \alpha$ ?

For finite $\alpha$, the answers to the four questions are affirmative and belong to the classical results of dimension theory (see for instance [1], §1.11). For infinite $\alpha$, questions 3 and 4 were asked e.g. by R. Engelking ([2], Problem 5.11), and the same set of questions were raised by L. Luxemburg ([11], Problem 8.4); question 1 was recently recalled in R. Pol's article [15] (Question $14.1(425))$.

Observe first that questions 2 and 4 are equivalent. This follows from the compactification theorem of L. Luxemburg (see [8], and [10] for the proof).

1.A. TheOREM ([8]). Every separable metrizable space $X$ has a metrizable compactification $Z$ such that Ind $Z=$ Ind $X$.

Now, let us state the results relating to our questions that can be found in the literature. Question 3 was answered in the affirmative by R. Pol:

1.B. Theorem ([14]). For every countable ordinal $\alpha$, there exists a universal space in the class of separable metrizable spaces $X$ with ind $X \leq \alpha$.

A partial positive result related to questions 1 and 2 can be found in R. Pol's paper [13]: for each countable ordinal $\alpha$, there exist a compact metrizable space $X_{\alpha}$ with ind $X_{\alpha}<\infty$ and a compact metrizable space $Y_{\alpha}$ with Ind $Y_{\alpha}<\infty$ such that $X_{\alpha}$ contains topologically all compact metrizable spaces $X$ with ind $X \leq \alpha$ and $Y_{\alpha}$ contains topologically all compact metrizable spaces $Y$ with Ind $Y \leq \alpha$.

On the other hand, some negative results concerning questions 1-4 were announced by L. Luxemburg (see [7]); to wit, for any infinite $\alpha$, there is no universal space in the class of all completely metrizable separable spaces $X$ such that ind $X \leq \alpha$ and $X$ is the union of a countable family of finitedimensional closed sets, nor in the class of all compact metrizable spaces $X$ 
such that ind $X \leq \alpha$ (Ind $X \leq \alpha$ ) and $X$ is the union of a countable family of finite-dimensional closed sets.

The present paper is devoted to the particular case of our questions, namely to the case when $\alpha=\lambda$ is a limit ordinal. We shall prove that in this case, the answers to questions 1 and 4, and thus also to question 2 , are negative.

Questions 1, 2 and 4 remain open for non-limit ordinals $\alpha \geq \omega$. We know neither the least possible transfinite dimension ind $X_{\lambda}$ of a compact metrizable space $X_{\lambda}$ containing topologically all compact metrizable spaces $X$ with ind $X=\lambda$, nor the least possible transfinite dimension Ind $Y_{\lambda}$ of a compact metrizable space $Y_{\lambda}$ containing topologically all compact metrizable spaces $Y$ with Ind $Y=\lambda$ (see [13], $\S 4,1$ ). The answers to these questions, even in the case $\lambda=\omega_{0}$, seem to have interesting applications in the theory of transfinite dimension.

Acknowledgements. The paper contains some of the results of my Ph.D. thesis supervised by Professor R. Engelking, whom I would like to thank for his comments and improvements. I am also grateful to Professor R. Pol who suggested the use of the spaces $C_{\lambda}^{\sigma}$ and $D_{\lambda}^{\sigma}$ (see Section 4) instead of the more complicated spaces originally used to the same end.

2. Smirnov's spaces. Yu. M. Smirnov [16] defined a sequence $\left\{S_{\alpha}\right.$ : $\left.\alpha<\omega_{1}\right\}$ of compact metrizable spaces. The definition is by transfinite induction on $\alpha: S_{0}$ is a one-point space; if $\alpha=\beta+1$ for a $\beta<\alpha$, then $S_{\alpha}=S_{\beta} \times I$; if $\alpha=\lambda$ is a limit ordinal, then $S_{\alpha}$ is the one-point compactification of the sum of topological spaces $\bigoplus\left\{S_{\beta}: \beta<\alpha\right\}$ (denote by $p_{\lambda}$ the unique point of the remainder). The space $S_{\omega_{0}+1}$ is exhibited in Fig. 2.1.

Yu. M. Smirnov evaluated the large transfinite dimension of $S_{\alpha}$.

2.A. Theorem ([16]). For every $\alpha<\omega_{1}$, we have Ind $S_{\alpha}=\alpha$.

Smirnov's spaces are also a source of examples of compact metrizable spaces with given small transfinite dimension. In order to get these examples, let us quote the following theorems.

2.B. Theorem ([6]). For every hereditarily normal compact space $X$, Ind $X \leq \omega_{0} \cdot$ ind $X$.

2.C. Theorem ([17]). For every metrizable space $X, \operatorname{Ind}(X \times I) \leq$ Ind $X+1$.

Combining Theorems 2.A-2.C and the obvious equality

$$
\text { ind } S_{\lambda}=\sup \left\{\text { ind } S_{\alpha}: \alpha<\lambda\right\},
$$

for every limit ordinal $\lambda<\omega_{1}$, we obtain the following corollary. 

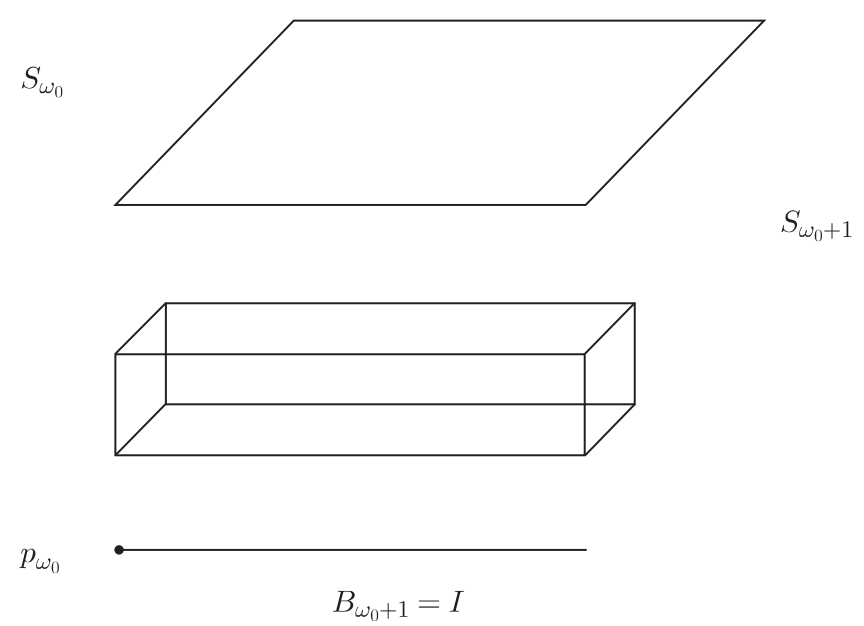

Fig. 2.1

COROllary. For every countable ordinal $\alpha$, there exists a countable ordinal $\beta \geq \alpha$ such that ind $S_{\beta}=\alpha$.

We denote by $\beta(\alpha)$ the smallest ordinal $\beta$ such that ind $S_{\beta}=\alpha$. Note that, as shown in [9], $\beta(\alpha)$ is greater than $\alpha$ for some ordinals $\alpha$, and ind $S_{\alpha}$ is unknown for some $\alpha$ (see [2], Problems 2.3 and 2.4). From (2.1) it follows immediately that

(2.2) if $\alpha$ is a non-limit ordinal, then so is $\beta(\alpha)$.

The rest of this section is devoted to a property of Smirnov's spaces (see Theorem 2.1) which will be of importance in the proofs of Theorems 5.1 and 5.2. In order to formulate and prove Theorem 2.1, we quote two results and introduce a notion.

The following lemma is a consequence of Lemma 7 of [16] and Lemma 1.2.9 of [1]; recall that if $\alpha=\lambda+n$, where $\lambda$ is a limit ordinal and $n$ is a natural number, then $S_{\alpha}=S_{\lambda} \times I^{n}$.

2.D. Lemma ([16]). Let $\alpha=\lambda+n$, where $\lambda$ is a limit ordinal and $n$ is a natural number. For every partition $L$ in $S_{\alpha}$ between $S_{\lambda} \times A$ and $S_{\lambda} \times B$, where $A, B$ is a pair of opposite $(n-1)$-dimensional faces of $I^{n}$, we have Ind $L \geq \lambda+(n-1)$.

Every non-limit ordinal $\alpha \geq \omega_{0}$ can be uniquely represented as the sum $\lambda+n$ of a limit ordinal $\lambda$ and a natural number $n$. From the construction of $S_{\alpha}$ it follows that $S_{\alpha}=S_{\lambda} \times I^{n}$. Denote by $B_{\alpha}$ the set $\left\{p_{\lambda}\right\} \times I^{n}$; we call $B_{\alpha}$ the base of $S_{\alpha}$. Sometimes, we will identify $B_{\alpha}$ and the $n$-dimensional cube 
$I^{n}$; in particular, we will write $S_{\alpha}=S_{\lambda} \times B_{\alpha}$. Let $B_{\alpha}=S_{\alpha}$ for $\alpha<\omega_{0}$, i.e., if $\alpha=n$, then $B_{\alpha}=S_{\alpha}=I^{n}$. Thus for every non-limit ordinal $\alpha<\omega_{1}$, the base of $S_{\alpha}$ is a finite-dimensional cube.

2.E. THEOREM ([17]). If a hereditarily normal space $X$ can be represented as the union $A_{1} \cup A_{2}$ of closed subspaces, and there is a homeomorphism $h: A_{1} \rightarrow A_{2}$ such that $h(x)=x$ for every $x \in A_{1} \cap A_{2}$, then ind $X=$ ind $A_{1}=$ ind $A_{2}$.

The above theorem appears in the section of [17] which considers only hereditarily normal spaces; one can verify, however, that the proof still goes when we only assume that $X$ is regular. In the present paper, we only need Theorem 2.E in the form given above.

In the proof of the following theorem and in the sequel, we will use the monotonicity of ind and of Ind with respect to closed subspaces (see [2], Proposition 3.3).

2.1. ThEOREM. Let $\alpha=\lambda+n$, where $\lambda$ is a countable limit ordinal, and $n$ is a natural number. For each partition $K$ in $S_{\alpha}$ between any distinct points $a, b \in B_{\alpha}$ we have ind $K \geq$ ind $S_{\alpha}-1$ and $\operatorname{Ind} K \geq \operatorname{Ind} S_{\alpha}-1=\lambda+(n-1)$.

Pr o of. We prove the first inequality, and then indicate the modifications needed to show the second.

First, we show that

$$
\operatorname{ind}_{x} S_{\alpha} \leq \text { ind } K+1 \quad \text { for each } x \in B_{\alpha},
$$

i.e., for every $x \in B_{\alpha}$ and each closed set $F \subseteq S_{\alpha}$ such that $x \notin F$ there exists a partition $L$ between $x$ and $F$ such that ind $L \leq$ ind $K$. The reasoning is divided into a few steps.

First, we show that

(2.4) if $x$ belongs to the geometrical boundary of $B_{\alpha}$, and $F$ is the union of $(n-1)$-dimensional faces of $B_{\alpha}$ not containing $a$, then there exists a partition with the required property.

Let $U$ and $V$ be disjoint open subsets of $S_{\alpha}$ such that $a \in U, b \in V$ and $K=S_{\alpha}-(U \cup V)$. Consider a subspace $Q \subseteq B_{\alpha}$ homeomorphic to the $n$-dimensional cube, and such that $a$ belongs to the geometrical boundary of $Q$ and the union $E$ of $(n-1)$-dimensional faces of $Q$ not containing $a$ is contained in $V$; then $K \cap\left(S_{\lambda} \times Q\right)$ is a partition in $S_{\lambda} \times Q$ between $a$ and $E$, and ind $K \cap\left(S_{\lambda} \times Q\right) \leq$ ind $K$. The existence of the required partition in $S_{\alpha}$ between $x$ and $F$ follows from the existence of a homeomorphism of $S_{\lambda} \times Q$ onto $S_{\alpha}=S_{\lambda} \times B_{\alpha}$ sending $a$ to $x$ and $E$ onto $F$.

In the second step, we show that the assumption that $x$ belongs to the geometrical boundary of $B_{\alpha}$ can be omitted in (2.4), i.e., 
(2.5) if $F$ is the union of the $(n-1)$-dimensional faces of $B_{\alpha}$ not containing $x$, then there exists a partition $L$ between $x$ and $F$ with ind $L \leq$ ind $K$.

Assume that $x=\left(p_{\lambda},\left(x_{1}, \ldots, x_{n}\right)\right)$ belongs to the geometrical interior of $B_{\alpha}=\left\{p_{\lambda}\right\} \times I^{n}$.

Let

$$
\begin{aligned}
& A_{1}=\left\{\left(z,\left(y_{1}, y_{2}, \ldots, y_{n}\right)\right) \in S_{\lambda} \times I^{n}: y_{1} \leq x_{1}\right\}, \\
& A_{2}=\left\{\left(z,\left(y_{1}, y_{2}, \ldots, y_{n}\right)\right) \in S_{\lambda} \times I^{n}: y_{1} \geq x_{1}\right\}
\end{aligned}
$$

further, let $h: S_{\alpha} \rightarrow S_{\alpha}$ be given by

$$
h\left(\left(z,\left(y_{1}, y_{2}, \ldots, y_{n}\right)\right)\right)=\left(z,\left(f\left(y_{1}\right), y_{2}, \ldots, y_{n}\right)\right),
$$

where

$$
f\left(y_{1}\right)= \begin{cases}\left(x_{1}-1\right) y_{1} / x_{1}+1 & \text { if } y_{1} \leq x_{1}, \\ x_{1}\left(y_{1}-1\right) /\left(x_{1}-1\right) & \text { if } y_{1} \geq x_{1}\end{cases}
$$

for $\left(z,\left(y_{1}, y_{2}, \ldots, y_{n}\right)\right) \in S_{\lambda} \times I^{n}=S_{\alpha}$.

Observe that $h$ is a homeomorphism of $S_{\alpha}$ mapping $A_{1}$ onto $A_{2}$ and such that $h(x)=x$ for every $x \in A_{1} \cap A_{2}$. By (2.4), there exists a partition $L_{1}$ in $A_{1}$ between $x$ and $F \cap A_{1}$ such that ind $L_{1} \leq$ ind $K$; let $L_{2}=h\left(L_{1}\right)$. It is easily seen that $L=L_{1} \cup L_{2}$ is a partition in $S_{\alpha}$ between $x$ and $F$; by Theorem 2.E, ind $L=$ ind $L_{1}$.

In the third step, we show that

for every closed set $F \subseteq B_{\alpha}$, there exists a partition $L$ between $x$ and $F$ such that ind $L \leq$ ind $K$.

Let $E$ be the union of $(n-1)$-dimensional faces of $B_{\alpha}$ not containing $x$. By (2.5), there exists a partition $M$ in $S_{\alpha}$ between $x$ and $E$ with ind $M \leq$ ind $K$; let $U$ and $V$ be disjoint open subsets of $S_{\alpha}$ such that $x \in U, E \subseteq V$ and $M=S_{\alpha}-(U \cup V)$.

Consider a homeomorphism $h: B_{\alpha} \rightarrow B_{\alpha}$ with $h(x)=x$ and $h(F) \subseteq$ $V \cap B_{\alpha}$; it can be defined by taking a neighbourhood of $x$ disjoint from $F$ and expanding it, without moving $x$, until it covers the set $B_{\alpha}-V$. Let $g: S_{\alpha} \rightarrow S_{\alpha}$ be given by $g(y, z)=(y, h(z))$ for $(y, z) \in S_{\lambda} \times B_{\alpha}=S_{\alpha}$. It is easy to observe that $L=g^{-1}(M)$ is a partition in $S_{\alpha}$ between $x$ and $F$, and ind $L=$ ind $M \leq$ ind $K$.

Having disposed of these preliminary steps, we can now prove (2.3).

Let $x \in B_{\alpha}$ be an arbitrary point and $F \subseteq S_{\alpha}$ an arbitrary closed set not containing $x$. Since $S_{\alpha}=B_{\alpha}$ for $\alpha<\omega_{0}$, we can assume that $\alpha \geq \omega_{0}$. Let $E=F \cap B_{\alpha}$ (see Fig. 2.2, where $\alpha=\omega_{0}+1$ ). By (2.6), there exists a partition $M$ between $x$ and $E$ such that ind $M \leq$ ind $K$; let $U, V \subseteq S_{\alpha}$ be disjoint open sets such that $x \in U, E \subseteq V$ and $M=S_{\alpha}-(U \cup V)$. 


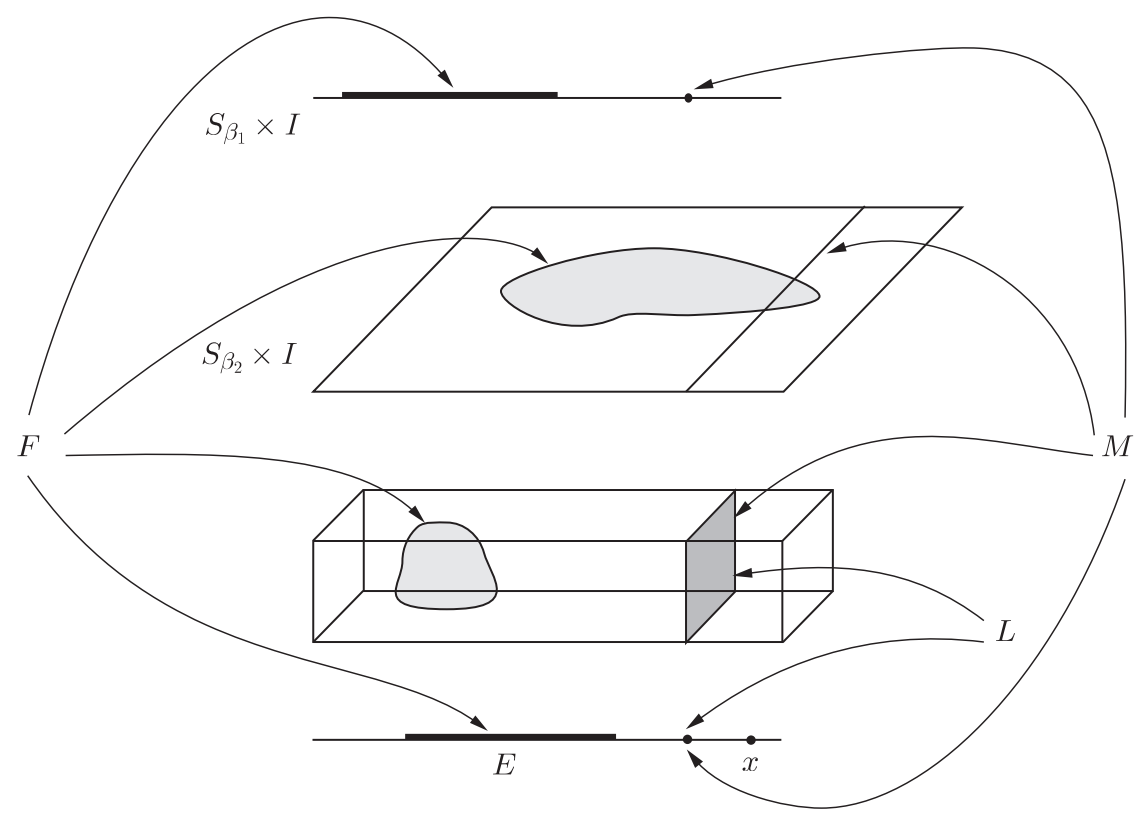

Fig. 2.2

By construction of $S_{\alpha}$, we have

$$
S_{\alpha}=B_{\alpha} \cup \bigcup\left\{S_{\beta} \times I^{n}: \beta<\lambda\right\} ;
$$

also, each compact subset of $S_{\alpha}$ disjoint from $B_{\alpha}$ meets only finitely many sets $S_{\beta} \times I^{n}$. Thus

$$
[F \cap(U \cup M)] \cap\left[S_{\beta} \times I^{n}\right] \neq \emptyset
$$

only for finitely many $\beta$; assume that the above intersection is empty for $\beta \neq \beta_{1}, \ldots, \beta_{k}$ (see Fig. 2.2, where $k=2$ ).

It is easily seen that $L=M-\bigcup_{i=1}^{k}\left(S_{\beta_{i}} \times I^{n}\right)$ is a partition between $x$ and $F$ (see Fig. 2.2), and ind $L \leq$ ind $M \leq$ ind $K$.

This proves (2.3). We now prove ind $S_{\alpha} \leq$ ind $K+1$ by induction on $\alpha$. For $\alpha<\omega_{0}$ the inequality is equivalent to (2.3); assume that $\alpha \geq \omega_{0}$ and the inequality holds for $\beta<\alpha$. By (2.3), we only have to show that $\operatorname{ind}_{x} S_{\alpha} \leq$ ind $K+1$ for every $x \in S_{\alpha}-B_{\alpha}$.

Observe that $K$ contains a partition in $S_{\beta} \times I^{n}=S_{\beta+n}$ between distinct points of the base $B_{\beta+n}$ for all but a finite number of ordinals $\beta<\lambda$. Thus, by the inductive assumption, ind $S_{\beta+n} \leq$ ind $K+1$ for those $\beta$. Since ind $S_{\nu} \leq$ ind $S_{\mu}$ whenever $\nu \leq \mu$, we have ind $S_{\beta+n} \leq$ ind $K+1$ for all $\beta<\lambda$; therefore every $x \in S_{\alpha}-B_{\alpha}$ has a neighbourhood $U$ in $S_{\alpha}$ with ind $U \leq$ ind $K+1$, which completes the proof of ind $S_{\alpha} \leq$ ind $K+1$.

We now sketch the proof of Ind $K \geq \lambda+(n-1)$. 
Of course, the inequality holds for $\alpha<\omega_{0}$; thus assume that $\alpha \geq \omega_{0}$. Let $A, B$ be a pair of opposite $(n-1)$-dimensional faces of $B_{\alpha}=I^{n}$. A reasoning similar to that in the proof of (2.4) shows that

there exists a partition $L$ in $S_{\alpha}$ between $A$ and $B$ such that Ind $L \leq$ Ind $K$.

Consider disjoint open sets $U, V \subseteq S_{\alpha}$ such that $A \subseteq U, B \subseteq V$, and $L=S_{\alpha}-(U \cup V)$. It follows immediately from the definition of $S_{\alpha}$ that there exist $\beta_{1}, \ldots, \beta_{k}<\lambda$ with the property that

$$
\begin{aligned}
& \left(\bigcup\left\{S_{\beta} \times I^{n}: \beta<\lambda \text { and } \beta \neq \beta_{1}, \ldots, \beta_{k}\right\}\right) \times A \subseteq U, \\
& \left(\bigcup\left\{S_{\beta} \times I^{n}: \beta<\lambda \text { and } \beta \neq \beta_{1}, \ldots, \beta_{k}\right\}\right) \times B \subseteq V .
\end{aligned}
$$

Let $f$ be a 1-1 mapping of the set of all ordinals $\beta<\lambda$ to the set of all ordinals $\beta<\lambda$ such that $\beta \neq \beta_{1}, \ldots, \beta_{k}$ with the property that $\beta \leq f(\beta)$ for every $\beta<\lambda$. Since $S_{\nu}$ is embeddable in $S_{\mu}$ whenever $\nu \leq \mu$, it follows that $S_{\beta}$ is embeddable in $S_{f(\beta)}$. Hence there exists an embedding of $S_{\lambda}$ in its subspace $\bigcup\left\{S_{\beta}: \beta<\lambda\right.$ and $\left.\beta \neq \beta_{1}, \ldots, \beta_{k}\right\}$, and so there also exists an embedding $h: S_{\alpha} \rightarrow S_{\alpha}$ such that

$$
\begin{aligned}
& h\left(S_{\lambda} \times A\right) \subseteq\left(\bigcup\left\{S_{\beta} \times I^{n}: \beta<\lambda \text { and } \beta \neq \beta_{1}, \ldots, \beta_{k}\right\}\right) \times A \subseteq U, \\
& h\left(S_{\lambda} \times B\right) \subseteq\left(\bigcup\left\{S_{\beta} \times I^{n}: \beta<\lambda \text { and } \beta \neq \beta_{1}, \ldots, \beta_{k}\right\}\right) \times B \subseteq V .
\end{aligned}
$$

The set $h^{-1}(L)$ is a partition in $S_{\alpha}$ between $S_{\lambda} \times A$ and $S_{\lambda} \times B$, and so, by Lemma 2.D,

$$
\lambda+(n-1) \leq \operatorname{Ind} h^{-1}(L) \leq \operatorname{Ind} L \leq \operatorname{Ind} K .
$$

3. Two lemmas. In this section we state two fairly straightforward, but important lemmas. They indicate a property of compact metrizable spaces $X$ with ind $X \leq \lambda$ and a property of separable metrizable spaces $X$ with Ind $X \leq \lambda$, respectively, which preclude the existence of universal spaces in these classes.

3.1. Lemma. Let $(X, \varrho)$ be a compact metric space with ind $X \leq \lambda$, where $\lambda$ is a limit ordinal, and let $\varepsilon$ be a positive real number. Then there exists an ordinal $\alpha<\lambda$ such that

for every subspace $A \subseteq X$ and for any $x, y \in A$ such that $\varrho(x, y) \geq \varepsilon$, there exists a partition in $A$ between $x$ and $y$ with small transfinite dimension not greater than $\alpha$.

P r o o f. Let $V_{1}, \ldots, V_{k}$ be a finite open covering of $X$ such that diam $V_{i}<$ $\varepsilon$ and ind bd $V_{i}<\lambda$ for $i=1, \ldots, k$; the existence of such a covering follows from the definition of ind and the compactness of $X$. 
Let $\alpha=\max \left\{\right.$ ind bd $\left.V_{i}: i=1, \ldots, k\right\}$.

Consider a subspace $A \subseteq X$ and points $x, y \in A$ such that $\varrho(x, y) \geq \varepsilon$. Then $x \in V_{i}$ for some $i$, and since $\operatorname{diam} V_{i}<\varepsilon$ and $\varrho(x, y) \geq \varepsilon$, we have $y \notin \operatorname{cl} V_{i}$. Thus $A \cap \operatorname{bd} V_{i}$ is a partition in $A$ between $x$ and $y$; obviously,

$$
\operatorname{ind}\left(A \cap \operatorname{bd} V_{i}\right) \leq \operatorname{ind} \text { bd } V_{i} \leq \alpha .
$$

3.2. Lemma. Let $(X, \varrho)$ be a totally bounded metric space with Ind $X \leq \lambda$, where $\lambda$ is a limit ordinal, and let $\varepsilon$ be a positive real number. Then there exists an ordinal $\alpha<\lambda$ such that

for every subspace $A \subseteq X$ and for any $x, y \in A$ such that $\varrho(x, y) \geq \varepsilon$, there exists a partition in $A$ between $x$ and $y$ with large transfinite dimension not greater than $\alpha$.

Proof. Let $W_{1}, \ldots, W_{k}$ be a finite open covering of $X$ such that $\operatorname{diam} W_{i}<\varepsilon$ for $i=1, \ldots, k$, and $F_{1}, \ldots, F_{k}$ its closed shrinking. Since Ind $X \leq \lambda$, it follows that for $i=1, \ldots, k$, there exists an open set $V_{i}$ such that $F_{i} \subseteq V_{i} \subseteq \operatorname{cl} V_{i} \subseteq W_{i}$ and Ind bd $V_{i}<\lambda$; obviously, $V_{1}, \ldots, V_{k}$ is also a finite open covering of $X$ and diam $\operatorname{cl} V_{i}<\varepsilon$ for $i=1, \ldots, k$.

A similar argument to that in the proof of Lemma 3.1 shows that $\alpha=$ $\max \left\{\right.$ Ind bd $\left.V_{i}: i=1, \ldots, k\right\}$ has the required property.

4. The compacta $C_{\lambda}^{\sigma}$ and $D_{\lambda}^{\sigma}$. Let $\lambda<\omega_{1}$ be a limit ordinal; denote by $\Lambda$ the set of all non-limit ordinals $\alpha$ such that $0<\alpha<\lambda$. In this section we associate with each sequence $\sigma: \mathbb{N} \rightarrow \Lambda$ compact metrizable spaces $C_{\lambda}^{\sigma}$ and $D_{\lambda}^{\sigma}$ such that ind $C_{\lambda}^{\sigma} \leq \lambda$ and Ind $D_{\lambda}^{\sigma} \leq \lambda$.

First, we define a certain subspace $Z$ of the square $I^{2}$.

Denote by $S_{n}$ the set of all pairs $(i, n)$, where $i=1,2, \ldots, 2^{n}$ and $n=$ $1,2, \ldots$, and put $S=\{0\} \cup \bigcup_{n=1}^{\infty} S_{n}$. Note that the sets $S_{n}$ are pairwise disjoint and $0 \notin S_{n}$ for all $n$.

Let $Z_{0}$ stand for the lower edge of $I^{2}$, i.e., the set of all $(x, y) \in I^{2}$ such that $y=0$; for $s=(i, n) \in S_{n}$, let $a_{s}$ denote the point $\left((2 i-1) / 2^{n+1}, 0\right) \in I^{2}$, i.e., the centre of the interval $\left\{(x, 0) \in I^{2}:(i-1) / 2^{n} \leq x \leq i / 2^{n}\right\}$ contained in the lower edge of $I^{2}$, and let $Z_{s}$ stand for the semicircle with centre $a_{s}$ and radius $1 / 2^{n+1}$ contained in our square, i.e., $Z_{s}=\left\{z \in I^{2}: \varrho\left(z, a_{s}\right)=\right.$ $\left.1 / 2^{n+1}\right\}$, where $\varrho$ denotes the standard metric on $I^{2}$.

Set $Z=\bigcup\left\{Z_{s}: s \in S\right\}$. It is a simple matter to check that $Z$ is a closed subspace of $I^{2}$. The space $Z$ is exhibited in Fig. 4.1.

Let us introduce the following notation: $z_{0}=(0,0), z_{1}=(1,0)$ and $z_{i}^{n}=\left(i / 2^{n}, 0\right)$ for $n \in \mathbb{N}$ and $i=0,1, \ldots, 2^{n}$. Since $z_{i}^{n}=z_{j}^{m}$ whenever $i / 2^{n}=j / 2^{m}$, and $z_{0}=z_{0}^{n}$ and $z_{1}=z_{2^{n}}^{n}$ for every $n \in \mathbb{N}$, some symbols stand for the same point, but that will cause no confusion. Note that $z_{i-1}^{n}, z_{i}^{n}$ are the endpoints of the $\operatorname{arc} Z_{s}$ for $s=(i, n) \in S_{n}$. 


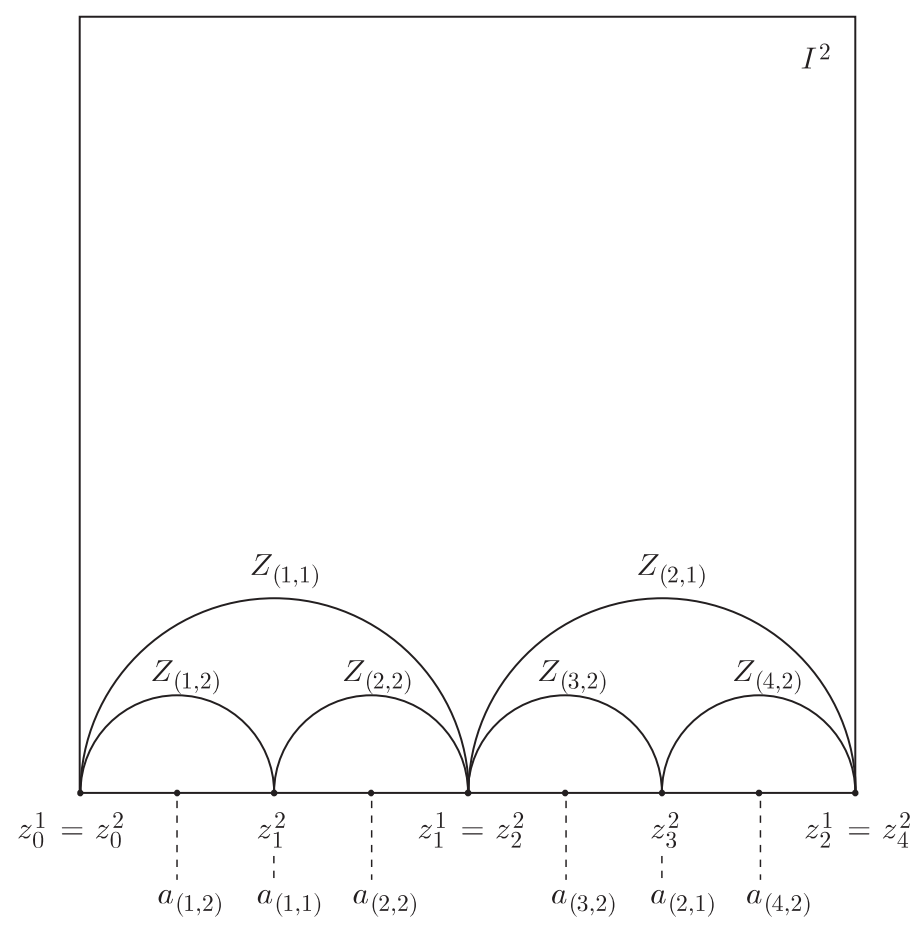

Fig. 4.1

We now pass to the construction of the spaces $C_{\lambda}^{\sigma}$ and $D_{\lambda}^{\sigma}$.

Let $S$ and $S_{n}$, for $n \in \mathbb{N}$, be the sets described in the definition of $Z$. Recall that $\beta(\alpha)$ denotes the smallest ordinal $\beta$ such that ind $S_{\beta}=\alpha$; since $\sigma(n) \in \Lambda$, it is a non-limit ordinal, and so is $\beta(\sigma(n))$ (see Section 2). Denote by $\gamma(n)$ the predecessor of $\beta(\sigma(n))$; by the definition of Smirnov's spaces, $S_{\beta(\sigma(n))}=S_{\gamma(n)} \times I$. Set $X_{s}=S_{\gamma(n)}$ and $b_{s}=p_{\gamma(n)}$ for $s \in S_{n}$, where $p_{\gamma(n)}$ is the distinguished point of $S_{\gamma(n)}$ (see Section 2). It will cause no confusion to identify $Z$ with the subspace of $Z \times \mathbb{P}\left\{X_{s}: s \in S-\{0\}\right\}$ consisting of all points $\left(x_{0},\left\{x_{s}: s \in S-\{0\}\right\}\right)$ such that $x_{s}=b_{s}$ for all $s \in S-\{0\}$. For $t \in S-\{0\}$, let $C_{t}$ be the subspace of the Cartesian product consisting of all $\left(x_{0},\left\{x_{s}: s \in S-\{0\}\right\}\right)$ such that $x_{0} \in Z_{t}$ and $x_{s}=b_{s}$ for $s \neq t$.

Note that since $Z_{t}$ is an arc, $C_{t}$ is homeomorphic to $S_{\gamma(n)} \times I=S_{\beta(\sigma(n))}$; moreover, there exists a homeomorphism of $C_{s}$ onto $S_{\beta(\sigma(n))}$ mapping $Z_{s}$ onto an edge of the base $B_{\beta(\sigma(n))}$.

Let $C_{\lambda}^{\sigma}=Z \cup \bigcup\left\{C_{t}: t \in S-\{0\}\right\}$. It is easily seen that $C_{\lambda}^{\sigma}$ is a closed subspace of $Z \times \mathbb{P}\left\{X_{s}: s \in S-\{0\}\right\}$, and therefore it is compact and metrizable.

Loosely speaking, in order to obtain $C_{\lambda}^{\sigma}$ we stick, for every $n \in \mathbb{N}$, a copy of $S_{\beta(\sigma(n))}$ to each arc $Z_{s}$, where $s \in S_{n}$, along an edge of the base $B_{\beta(\sigma(n))}$ in such a way that the space $C_{\lambda}^{\sigma}$ so obtained is compact, i.e., the diameters of the stuck copies of Smirnov's spaces converge to 0 as $n$ tends to $\infty$. 
The space $D_{\lambda}^{\sigma}$ is defined similarly, except that $S_{\beta(\sigma(n))}$ is replaced by $S_{\sigma(n)}$; recall that Ind $S_{\sigma(n)}=\sigma(n)$.

The space $C_{\lambda}^{\sigma}$, where $\lambda=\omega_{0}$ and $\sigma$ given by $\sigma(n)=n+1$ for every $n \in \mathbb{N}$, is exhibited in Fig. 4.2. Note that if $\lambda=\omega_{0}$, then $C_{\lambda}^{\sigma}=D_{\lambda}^{\sigma}$ for every $\sigma$.

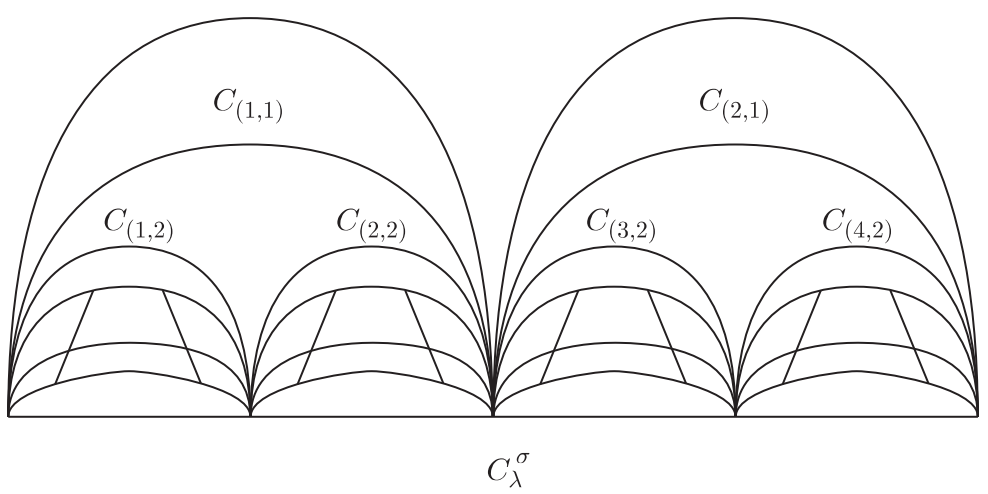

Fig. 4.2

We now evaluate ind $C_{\lambda}^{\sigma}$ and Ind $D_{\lambda}^{\sigma}$. To this end, we need the following theorem which is a consequence of a theorem obtained independently by M. Landau and A. R. Pears (see [5] and [12], and also [2], Theorem 5.17 ); it also follows from a theorem of B. T. Levshenko (see [6], and [2], Theorem 5.15).

4.A. Theorem ([5], [12] and [6]). If a hereditarily normal space $X$ can be represented as the union $A_{1} \cup A_{2}$ of its closed subspaces with Ind $A_{1}<\lambda$ and Ind $A_{2}<\lambda$, where $\lambda$ is a limit ordinal, then Ind $X<\lambda$.

4.1. Lemma. For every limit ordinal $\lambda<\omega_{1}$ and every sequence $\sigma$ : $\mathbb{N} \rightarrow \Lambda$, we have ind $C_{\lambda}^{\sigma} \leq \lambda$ and Ind $D_{\lambda}^{\sigma} \leq \lambda$.

Proof. We show that $\operatorname{ind}_{z} C_{\lambda}^{\sigma} \leq \lambda$ for every $z \in C_{\lambda}^{\sigma}$.

Note that $C_{\lambda}^{\sigma}=Z_{0} \cup \bigcup\left\{C_{s}-Z_{0}: s \in S-\{0\}\right\}$. Since $C_{s}-Z_{0}$ is an open subset of $C_{\lambda}^{\sigma}$ and ind $\left(C_{s}-Z_{0}\right) \leq \operatorname{ind} C_{s}<\lambda$, it follows that $\operatorname{ind}_{z} C_{\lambda}^{\sigma}<\lambda$ for every $z \in C_{\lambda}^{\sigma}-Z_{0}$.

Thus consider a $z \in Z_{0}$. Assume first that $z \neq z_{i}^{n}$ for any $n \in \mathbb{N}$ and $i=1,2, \ldots, 2^{n}$. Let $A \subseteq C_{\lambda}^{\sigma}$ be a closed set such that $z \notin A$. Take $n \in \mathbb{N}$ and $i \leq 2^{n}$ with the property that $x$ belongs to the segment with endpoints $z_{i-1}^{n}$ and $z_{i}^{n}$ contained in $Z_{0}$, and $A$ meets neither this segment nor any $C_{s}$, where $s \in \bigcup\left\{S_{m}: m \geq n\right\}$, such that the endpoints of $Z_{s}$ belong to this segment. One can readily check that $L=\left\{z_{i-1}^{n}, z_{i}^{n}\right\}$ is a partition between $z$ and $A$ (see Fig. 4.3). 


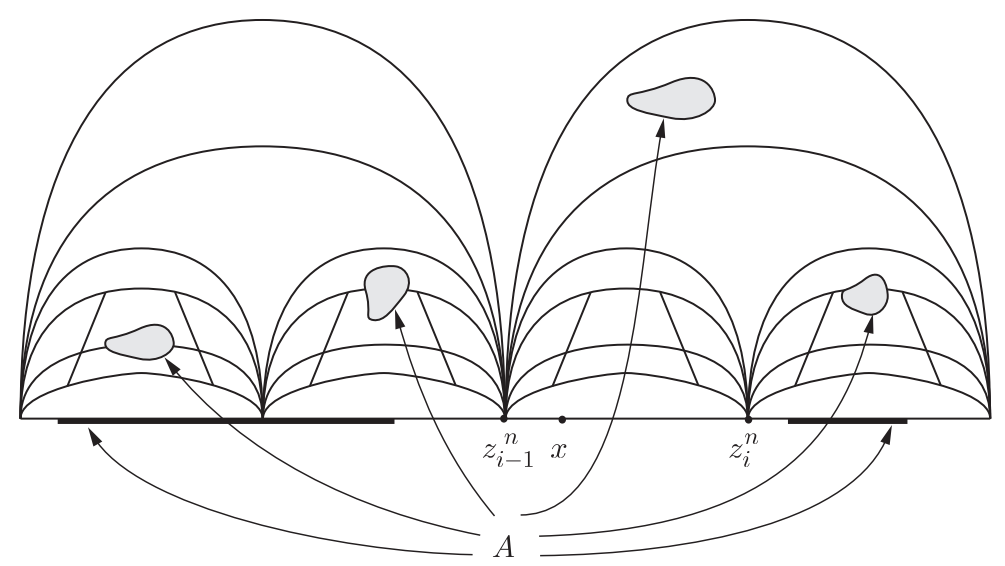

Fig. 4.3

Suppose now that $z=z_{i}^{n}$ for some $n \in \mathbb{N}$ and $i \leq 2^{n}$; obviously, one can assume that $n$ is the smallest number for which there exists an $i \leq 2^{n}$ such that $z=z_{i}^{n}$. Let $A \subseteq C_{\lambda}^{\sigma}$ be a closed set such that $z \notin A$. Then there exist $m>n$ and $j \leq 2^{m}$ with $z_{i}^{n}=z_{j}^{m}$, and $A$ meets neither the segment with endpoints $z_{j-1}^{m}$ and $z_{j+1}^{m}$ contained in $Z_{0}$ nor any $C_{s}$, where $s \in \bigcup\left\{S_{k}: k>m\right\}$, such that the endpoints of $Z_{s}$ belong to this segment. For every $k \in\{n, n+1, \ldots, m\}, z=z_{i}^{n}$ is an endpoint of $Z_{s}$ for two $s \in S_{k}$; denote by $z_{s}$ the other endpoint of $Z_{s}$. Let $L_{k}^{1}$ be a partition in $C_{s}$ between $z_{i}^{n}$ and $\left(A \cap C_{s}\right) \cup\left\{z_{s}\right\}$ such that ind $L_{k}^{1}<\lambda$ for one of these two indices $s$, and $L_{k}^{2}$ a partition with the same properties for the other one. It followsimmediately that $L=\left\{z_{j-1}^{m}, z_{j+1}^{m}\right\} \cup \bigcup_{k=n}^{m}\left(L_{k}^{1} \cup L_{k}^{2}\right)$

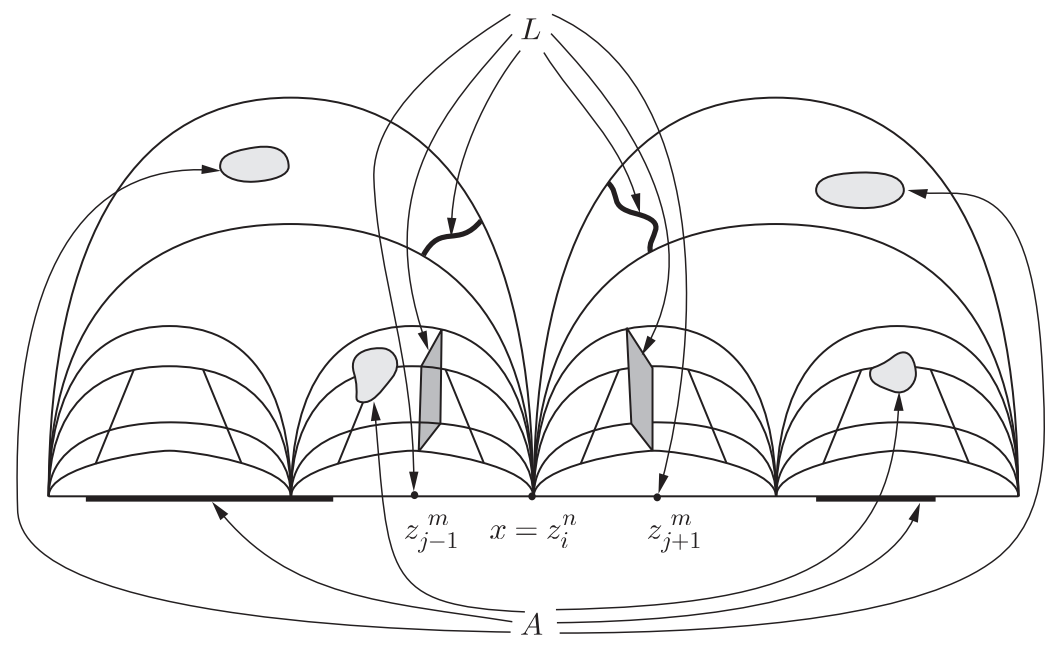

Fig. 4.4 
is a partition in $C_{\lambda}^{\sigma}$ between $z=z_{i}^{n}$ and $A$ (see Fig. 4.4). Since the sets $\left\{z_{j-1}^{m}, z_{j+1}^{m}\right\}, L_{n}^{1}, L_{n}^{2}, L_{n+1}^{1}, L_{n+1}^{2}, \ldots, L_{m}^{1}, L_{m}^{2}$ are pairwise disjoint, compact and have small transfinite dimension less than $\lambda$, we have ind $L<\lambda$.

Thus ind $C_{\lambda}^{\sigma} \leq \lambda$. In order to prove Ind $D_{\lambda}^{\sigma} \leq \lambda$, observe that a similar reasoning shows that for every $z \in D_{\lambda}^{\sigma}$ and every closed set $B \subseteq D_{\lambda}^{\sigma}$ such that $z \notin B$, there exists a partition $L$ between $z$ and $B$ with Ind $L<\lambda$.

Let $A$ be a closed subset of $D_{\lambda}^{\sigma}$ and $V$ an open set containing $A$. For every $z \in A$, consider a partition $L_{z}$ between $z$ and $D_{\lambda}^{\sigma}-V$ with Ind $L_{z}<\lambda$; obviously, one can assume that $L_{z}=$ bd $W_{z}$ for some neighbourhood $W_{z} \subseteq$ $V$ of $z$. By compactness of $A$, there exists a finite family $\mathcal{W} \subseteq\left\{W_{z}: z \in\right.$ $A$ \} such that $A \subseteq \bigcup \mathcal{W}$; let $U=\bigcup \mathcal{W}$. Then $A \subseteq U \subseteq V$ and bd $U \subseteq$ $\bigcup\{\operatorname{bd} W: W \in \mathcal{W}\}$; in particular, Ind bd $U \leq \operatorname{Ind}(\bigcup\{\operatorname{bd} W: W \in \mathcal{W}\})$. Now Theorem 4.A shows that Ind bd $U<\lambda$.

5. There is no universal space for spaces with limit transfinite dimension. In this section we prove the theorems announced in Section 1.

5.1. Theorem. Let $\lambda<\omega_{1}$ be an arbitrary limit ordinal. There is no universal space for compact metrizable spaces $X$ with ind $X \leq \lambda$.

P r o of. It suffices to show that

(5.1) for every compact metrizable space $X$ with ind $X \leq \lambda$, there exists a sequence $\sigma: \mathbb{N} \rightarrow \Lambda$ such that $C_{\lambda}^{\sigma}$ is not homeomorphic to any subspace of $X$.

Let $\varrho$ be an arbitrary metric on $X$. It follows from Lemma 3.1 that for every $n \in \mathbb{N}$, there exists an ordinal $\alpha_{n}<\lambda$ satisfying (3.1) for $\varepsilon=2^{-2 n}$. Set $\sigma(n)=\alpha_{n}+2$ for $n \in \mathbb{N}$. Since $\lambda$ is a limit ordinal, $\sigma(n)<\lambda$ for each $n \in \mathbb{N}$, and therefore $\sigma(n) \in \Lambda$ for every $n \in \mathbb{N}$. Suppose that there exists an embedding $h: C_{\lambda}^{\sigma} \rightarrow X$.

Since there exists a homeomorphism of $C_{s}, s \in S_{n}$, onto $S_{\beta(\sigma(n))}$ mapping $Z_{s}$ onto an edge of the cube $B_{\beta(\sigma(n))} \subseteq S_{\beta(\sigma(n))}$, there is no partition in $C_{s}$ between any two distinct points of $Z_{s}$ with small transfinite dimension less than the predecessor of $\sigma(n)$ (see the first inequality of Theorem 2.1). Thus there is no partition in $h\left(C_{s}\right)$ between any two distinct points of $h\left(Z_{s}\right)$ with small transfinite dimension not greater than $\alpha_{n}$. From (3.1), we have $\operatorname{diam} h\left(Z_{s}\right)<2^{-2 n}$.

Hence, by the triangle inequality,

$$
\begin{aligned}
\varrho\left(h\left(z_{0}\right), h\left(z_{1}\right)\right) & \leq \sum_{i=1}^{2^{n}} \varrho\left(h\left(z_{i-1}^{n}\right), h\left(z_{i}^{n}\right)\right) \\
& \leq \sum_{s \in S_{n}} \operatorname{diam} h\left(Z_{s}\right)<2^{n} \cdot 2^{-2 n}=2^{-n}
\end{aligned}
$$


recall that $z_{0}$ and $z_{1}$ are the endpoints of the interval $Z_{0} \subseteq Z$, and $\left\{z_{i}^{n}: i=\right.$ $\left.1,2, \ldots, 2^{n}\right\}$ stands for the sequence of points of $Z$ with the property that $z_{i-1}^{n}, z_{i}^{n}$ are the endpoints of the arc $Z_{s}$ for $s=(i, n) \in S_{n}$.

Since $n$ is an arbitrary natural number, we conclude that $h\left(z_{0}\right)=h\left(z_{1}\right)$, which contradicts the assumption that $h$ is a homeomorphism.

5.2. Theorem. Let $\lambda<\omega_{1}$ be an arbitrary limit ordinal. There is no universal space for separable metrizable spaces $X$ with $\operatorname{Ind} X \leq \lambda$.

P r o of. It suffices to show that

(5.2) for every separable metrizable space $X$ with Ind $X \leq \lambda$, there exists a sequence $\sigma: \mathbb{N} \rightarrow \Lambda$ such that $D_{\lambda}^{\sigma}$ is not homeomorphic to any subspace of $X$.

To this end, take a totally bounded metric $\varrho$ on $X$ and apply a reasoning similar to that in the proof of Theorem 5.1 using Lemma 3.2 instead of Lemma 3.1 and the second inequality of Theorem 2.1 instead of the first one.

\section{References}

[1] R. Engelking, Dimension Theory, PWN, Warszawa, 1978.

[2] —, Transfinite dimension, in: Surveys in General Topology, G. M. Reed (ed.), Academic Press, 1980, 131-161.

[3] —, General Topology, Heldermann, Berlin, 1989.

[4] W. Hurewicz, Ueber unendlich-dimensionale Punktmengen, Proc. Akad. Amsterdam 31 (1928), 167-173.

[5] M. Landau, Strong transfinite ordinal dimension, Bull. Amer. Math. Soc. 21 (1969), 591-596.

[6] B. T. Levšenko [B. T. Levshenko], Spaces of transfinite dimensionality, Mat. Sb. 67 (1965), 255-266 (in Russian); English transl.: Amer. Math. Soc. Transl. Ser. 2 73 (1968), 135-148.

[7] L. A. Luxemburg, On infinite-dimensional spaces with transfinite dimension, Dokl. Akad. Nauk SSSR 199 (1971), 1243-1246 (in Russian); English transl.: Soviet Math. Dokl. 12 (1971), 1272-1276.

[8] - On transfinite inductive dimensions, Dokl. Akad. Nauk SSSR 209 (1973), 295298 (in Russian); English transl.: Soviet Math. Dokl. 14 (1973), 388-393.

[9] L. A. Luxemburg, On compact spaces with non-coinciding transfinite dimensions, Dokl. Akad. Nauk SSSR 212 (1973), 1297-1300 (in Russian); English transl.: Soviet Math. Dokl. 14 (1973), 1593-1597.

[10] - On compactifications of metric spaces with transfinite dimensions, Pacific J. Math. 101 (1982), 399-450.

[11] -, On universal infinite-dimensional spaces, Fund. Math. 122 (1984), 129-147.

[12] A. R. Pears, A note on transfinite dimension, ibid. 71 (1971), 215-221.

[13] R. Pol, On classification of weakly infinite-dimensional compacta, ibid. 116 (1983), 169-188. 
[14] R. Pol, Countable-dimensional universal sets, Trans. Amer. Math. Soc. 297 (1986), 255-268.

[15] -, Questions in dimension theory, in: Open Problems in Topology, J. van Mill and G. M. Reed (eds.), North-Holland, 1990, 279-291.

[16] Yu. M. Smirnov, On universal spaces for certain classes of infinite dimensional spaces, Izv. Akad. Nauk SSSR Ser. Mat. 23 (1959), 185-196 (in Russian); English transl.: Amer. Math. Soc. Transl. Ser. 221 (1962), 21-33.

[17] G. H. Toulmin, Shuffling ordinals and transfinite dimension, Proc. London Math. Soc. 4 (1954), 177-195.

DEPARTMENT OF MATHEMATICS

UNIVERSITY OF WARSAW

BANACHA 2

02-097 WARSZAWA, POLAND

Received 24 February 1993;

in revised form 3 June 1993 